\title{
On the Study of Wireless Signal Noise for Designing Network Infrastructure of Knowledge Management Systems
}

\author{
Chunbo Luo ${ }^{\dagger}$, Pablo Casaseca ${ }^{\ddagger}$, Xinheng Wang ${ }^{\ddagger}$, Keshav Dahal ${ }^{\ddagger}$, Cheng Jin\#, Peng Ren ${ }^{\$}$ \\ $†$ College of Engineering, Mathematics and Physical Sciences, University of Exeter, Exeter, EX4 4QF, UK \\ $\ddagger$ School of Engineering\&Computing, University of the West of Scotland, Paisley, PA1 2BE, UK \\ \# Beijing Institute of Technology, Beijing, 100081, China \\ $\$$ China University of Petroleum(Huadong), Qingdao, 266580, China
}

\begin{abstract}
Knowledge and information management systems are usually supported by wireless networks that strongly rely on reliable received signal strength. The interruption and outage of such system may lead to significant performance disruption. In order to deal with one of the major contributors: noise, this paper investigates the fundamentals of wireless signals and proposes a method to identify and model the noise components quantitatively. We investigate the theoretical method and empirically study two wireless system configurations - one with omnidirectional antennas and one with directional antennas. Results based on real-world experiments confirm the existence and exact contributions of coloured noise components. Based on the preliminary results of this study, future information management systems can be designed with enhanced network support to cope with the variation of signals for improved performance.

Index Terms-Information management system, wireless signal, coloured noise, modelling, Allan variance
\end{abstract}

\section{INTRODUCTION}

The convergence of knowledge and information management systems with networks has significant potential to provide services anytime and anywhere. However, the performance of a system can be severely affected if the network cannot provide sufficient bandwidth and reliability. Networks often utilize received signal strength (RSS) as a performance index because its value indicates the signal quality and suggests whether the system can properly function or not [1]-[4]. For example, a management system with inaccurate RSS may lead to the failure in sending important commands or the loss of key data. Such problem becomes more severe with the prevalence of wireless networks. Because of the dynamic propagation features of radio frequency channels and transceiver electronic system noise, we can observe that RSS measurements vary irregularly in practical scenarios. Therefore the accurate identification and modelling of RSS noise components is an important topic. ${ }^{1}$

The Friis transmission equation can be used to characterise and model the received wireless signal strength

\footnotetext{
${ }^{1}$ This paper is sponsored by the Research Councils UK Digital Economy Theme Sustainable Society Network+ and Royal Society-NSFC Grant No. IE131036, and partially supported by DHI Scotland through the Smartcough/Macmasters project.
}

under ideal conditions of free space [5], however, the practical propagation environment where wireless signals are generated and propagated is usually far from ideal. In [6], the authors modelled RSS as the combined effect of three major factors: antenna gain, transmitting power and free space path loss, with an additional loss factor to capture randomness. This random factor was modelled to follow a white Gaussian process with zero mean: the additional white noise in Friis equation [7], [8]. This model has been broadly accepted and applied in communications and wireless networks [9]-[11].

If the noise power approximately equally spreads the whole bandwidth, such assumption fits the practical case very well. However, this noise type cannot characterise the components with different power spectral densities (PSD). For example, the noise associated to some physical instruments has both short and long term correlations due to circuit drifts, and the corresponding components thus have different PSDs [12]. The use of a white process to model noise may oversimplify the problem. The noise components which do not equally span over the full bandwidth can be named as coloured noise, which is commonly found in systems such as measuring instruments [12]. From this observation, it is necessary to identify different noise types, model them and reduce their malignant effects when designing future knowledge management systems.

This paper first demonstrates the time and systemdependency features of RSS measurements from practical system set-ups. As an effective instrument to analyze coloured noises, Allan variance (AVAR) is then introduced with the focus on characterising different types of noise [13]. Theoretical analysis shows that RSS noises have exponential identities and can be processed explicitly if they are handled correctly. In order to apply the modelling method in practical applications and utilize RSS values without considering every potential component individually, we highlight the coloured noise components from the RSS measurements with the focus on their corresponding parameters, which are obtained through Least Squares (LS). Two typical experiments - one from an omnidirectional antenna and another from a directional one - are conducted 
and analyzed. The obtained results can be used as references and guidance for designing knowledge management systems.

The remainder of the paper is as follows: Section II discusses RSS modelling, and the calculation method for AVAR and noise components; Section III presents two different experiments based on wireless systems with omnidirectional and directional antennas respectively; Section IV discusses the relevant issues of RSS measurement and noise modelling; Section V concludes this paper.

\section{RSS NOISE ANALYSIS}

\section{A. RSS and Time Dependency}

A wireless system generates signals using its internal circuit and transmits them from its antennas. The radio frequency signals are broadcast through propagation medium using electromagnetic waves. The major causes to the degradation of the received signal strength include thermal noise, antenna features and channel propagation loss. According to [7], the received signal strength can be given by

$$
P_{y}(d)[d B]=P_{x}-\bar{P} L\left(d_{0}\right)-10 n \log \left(\frac{d}{d_{0}}\right)+X_{\sigma} .
$$

where $n$ is the path loss factor whose value is associated with the propagation environment [9], $P_{x}$ is the transmitting power, $d$ and $d_{0}$ are the transmitter-receiver distance and reference distance respectively. As described before, $X_{\sigma}$ is the random noise term. Given a fixed position of transmitter and receiver, we can rewrite (1) to describe the time varying relationship as

$$
P_{y}(t)[d B]=P_{x}-\bar{P} L\left(d_{0}\right)-10 n \log \left(\frac{d}{d_{0}}\right)+X_{\sigma}(t),
$$

where the first three terms on the right hand side are not time varying.

In the literature (e.g. [6], [14]), $X_{\sigma}(t)$ is modelled as a zero-mean Gaussian distributed random variable (in $\mathrm{dB}$ ) with standard deviation $\sigma$ and its power spectral density is given by

$$
S_{w}(f)=\frac{N_{0}}{2}
$$

where $N_{0}=2 \sigma^{2}$.

Using a white process to model the random variable is convenient in describing its movements driven by random forces and has the advantage of simplicity in mathematical expression and analysis. However, it can be an oversimplified makeshift in describing practical systems where the noise is due to complicated sources and shows different behaviours at different correlation times [15]. In order to verify that the noise present in RSS measurements is actually coloured, we tested a dataset of $N=15000$ RSS measurements from a $802.11 \mathrm{~g}$ receiver (The experimental conditions are given in Section III-A.) against the null hyphotesis of whiteness of the noise. We employed the LjungBox Q-test [16] for the sample autocorrelation coefficient of the noise process $X_{\sigma}(j), j=1, \ldots, N$ :

$$
\hat{\rho}(k)=\frac{\sum_{j=k+1}^{N} X_{\sigma}(j) X_{\sigma}(j-k)}{\sum_{j=1}^{N} X_{\sigma}^{2}(j)} \quad k \in \mathbb{Z}
$$

with null and alternative hypothesis:

$$
\begin{gathered}
\mathcal{H}_{0}(\text { white noise }): \hat{\rho}(k)=0 \quad \forall k \neq 0 \\
\mathcal{H}_{1}(\text { coloured noise }): \hat{\rho}(k) \neq 0 \quad \text { for some } k \neq 0 .
\end{gathered}
$$

Under the null hypothesis, the statistic

$$
Q_{m}=N(N+2) \sum_{k=1}^{m} \frac{\hat{\rho}^{2}(k)}{N-k}
$$

asymptotically follows a Chi-squared distribution with $m$ degrees of freedom $\left(\chi_{m}^{2}\right)$. Figure 1 shows the obtained $Q_{m}$ values for $m=1,2, \ldots, 100$ together with the threshold values at which, we would reject the null hypothesis at $\alpha=$ 0.05 significance level. The obtained values are far above the thresholds which means that the null hypothesis can be rejected with a very low error probability. Actually, the $p$ values obtained from our data are all below $2.22 \cdot 10^{-16}$ (MATLAB floating point accuracy).

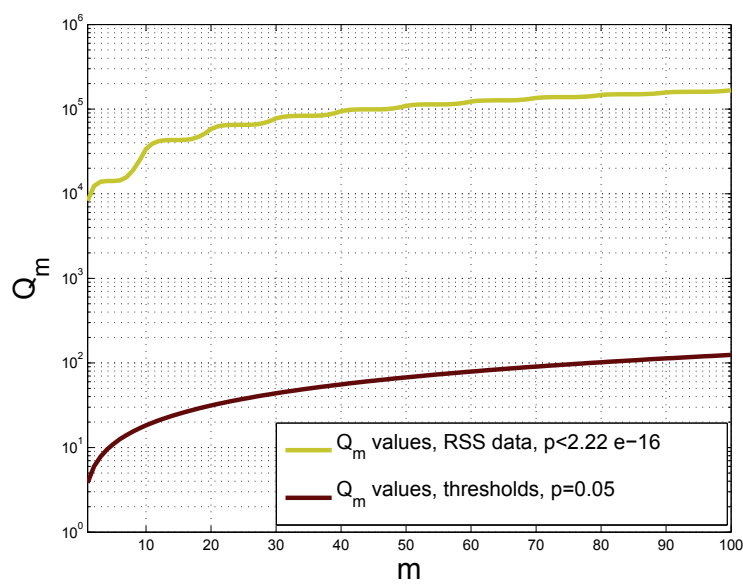

Fig. 1. Ljung-Box Q-test statistics obtained for $m=1,2, \ldots, 100$ time lags from $N=15000$ RSS samples. Threshold values for $\alpha=0.05$ are also presented

The results of this test show that the noise present in the RSS measurements has a time-dependant statistical behaviour. In order to analyze this noise, the instrument of AVAR will be introduced as an effective tool to identify the noise components.

\section{B. Allan Variance}

Allan variance (AVAR) was proposed by David W. Allan to characterise the underlying noise processes of clock systems in 1966 [13] and, together with some modifications, it has been recommended as a standard for such purpose [17]. Let $X(t)$ be the noise signal under study. The AVAR 
is obtained for different time stamps $t_{m}$ as:

$\sigma_{\tau}^{2}=\frac{1}{2} \operatorname{Var}\left\{\bar{X}\left(t_{m}+\tau\right)-\bar{X}\left(t_{m}\right)\right\}=\frac{1}{2} \operatorname{Var}\left\{\bar{X}_{m+1}-\bar{X}_{m}\right\}$

where

$$
\bar{X}_{m}=\frac{1}{\tau} \int_{t_{m}}^{t_{m}+\tau} X(t) d t
$$

and, for stationary processes, is only a function of $\tau$. With this in mind, the AVAR can be easily estimated by numerically computing (4) as a sample average for different time instants $t_{m}, m=1, \ldots, M-1$ and the AVAR as a sample variance (note that $E\left\{\bar{X}_{m+1}-\bar{X}_{m}\right\}=0$ for stationary processes:

$$
\hat{\sigma}_{\tau}^{2}=\frac{1}{2(M-1)} \sum_{m=1}^{M-1}\left(\mu_{m+1}-\mu_{m}\right)^{2}
$$

where

$$
\mu_{m}=\frac{1}{K} \sum_{k=1}^{K} X\left(t_{m}+k T s\right)
$$

with $T_{s}$ the sampling time and $K=\left\lfloor\tau / T_{s}\right\rfloor$. The number of available points $M$ to calculate the sample variance is a function of the total length of the signal $N \cdot T_{s}$ and the integration interval $\tau$ :

$$
M=\left\lfloor\frac{N T_{s}}{\tau}\right\rfloor
$$

An example figure can be seen in Fig.3.

It is worth noting here that the error of the AVAR estimator, usually decreases as the averaging time $\tau$ increases. Confidence intervals for the estimation can be established by considering the distribution of the sample variance. Thus, we can use the Chi-squared distribution to establish its confidence interval as follows

$$
\chi^{2}=\frac{\digamma \hat{\sigma}_{\tau}^{2}}{\sigma_{\tau}^{2}},
$$

where $\sigma_{\tau}^{2}$ is the true variance value and $\digamma$ is the number of degrees of freedom (DOF) of the estimator ${ }^{2} \cdot \chi^{2}$ denotes the cumulative distribution function of the chi-squared distribution. Given a confidence of $\varepsilon$, the confidence interval for the Allan variance estimation is given by

$$
\frac{\digamma \hat{\sigma}_{\tau}^{2}}{\chi^{2}(\varepsilon)} \leq \sigma_{\tau}^{2} \leq \frac{\digamma \hat{\sigma}_{\tau}^{2}}{\chi^{2}(1-\varepsilon)} .
$$

Because the noise term $X_{\sigma}(t)$ in (2) is composed of multiple noise components, it is important to know the contribution of each component and model these noise given its contributing terms. Therefore, we apply the AVAR tool in this paper in order to not only obtain their contributions but also to apply them in practice. Let us consider a (one-sided) power-law spectral density (PSD) which can be reasonably used to model the random fluctuations in RSS signals (see

\footnotetext{
${ }^{2}$ The number of DOF should be specifically estimated since the samples used for computing AVAR are seldom uncorrelated.
}

Section II-C):

$$
S_{X}(f)=\sum_{\alpha} h_{\alpha} f^{\alpha},
$$

In practice, these random fluctuations can often be represented by the sum of five noise processes $-2 \leq \alpha \leq 2$ assumed to be independent [13], [17]. Specifically, for the model in (6), and considering the bounds for $\alpha$, the timedomain measure can be expressed as:

$$
\sigma_{\tau}^{2} \approx A h_{-2} \tau+B h_{-1}+\frac{C h_{0}}{\tau}+\frac{D h_{1}+E h_{2}}{\tau^{2}}
$$

with the mapping coefficients given by

$$
\begin{aligned}
& A=\frac{2 \pi^{2}}{3}, B=2 \ln (2), C=\frac{1}{2}, \\
& D=\frac{1.038+3 \ln \left(w_{h} \tau\right)}{4 \pi^{2}}, E=\frac{3 f_{h}}{4 \pi^{2}},
\end{aligned}
$$

where $f_{h}=w_{h} /(2 \pi)$ is the bandwidth of the measurement system.

The $h_{-\alpha}$ coefficients can be estimated using Least Squares (LS) algorithms [18]. The introduction of AVAR to characterise the noise PSD may be preferable to (for instance) directly fitting (6) using the periodogram. To illustrate this, we conducted a simple experiment: We generated 1000 realizations (where each one has $N=2^{14}=16384$ samples) of a noise process whose power spectral density is given by (6) with $h_{-2}=0.01, h_{-1}=1, h_{0}=100, h_{1}=$ $0, h_{2}=0$ as in [18]. For each of them, we estimated the two sets of $h_{\alpha}$ values using LS to fit the AVAR curve $\left(\hat{h}_{\alpha}^{\sigma}\right)$ and the PSD directly estimated with the periodogram $\left(\hat{h}_{\alpha}^{P S D}\right)$, respectively, for the sake of comparison. The histograms for the relative errors $e_{\alpha}^{i}=\left(\hat{h}_{\alpha}^{i}-h_{\alpha}\right) / h_{\alpha}$ are presented in Figure 2, where lower values and dispersion from the AVAR estimations can be easily observed.

\section{Noise Components and Sources}

As described in [13], coloured noise is a combination of several types of components which cover different frequency bands of the noise spectrum. Similar to [13] and the IEEE standard [17], we only consider the major components which contribute the most part of the noise spectrum and AVAR values. Specifically the following 5 components: $-2 \leq \alpha \leq 2$ are analyzed. Some details of these noise components are introduced below [19]:

- Brow(nian) noise $(\alpha=-2)$ : This type of noise corresponds to a random walk behavior of the received signal [8] . Its origin is actually Brownian motion in the receiver circuitry.

- Pink noise $(\alpha=-1)$ : This component, also referred to as flicker noise, shows a $1 / f$ pattern. It has a variety of different causes, usually related to the flow of direct current.

- White noise $(\alpha=0)$ : Named by analogy to white light, with a flat frequency spectrum. As a wideband noise, RSS measurements have the equivalent component of white noise denoted by (2). The source of this 


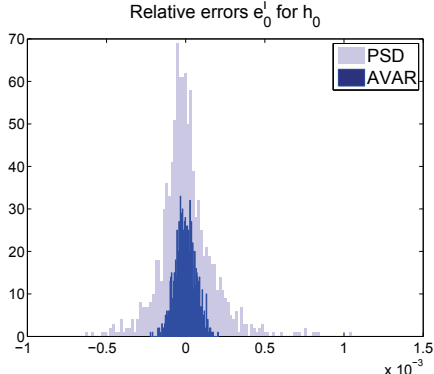

(a)

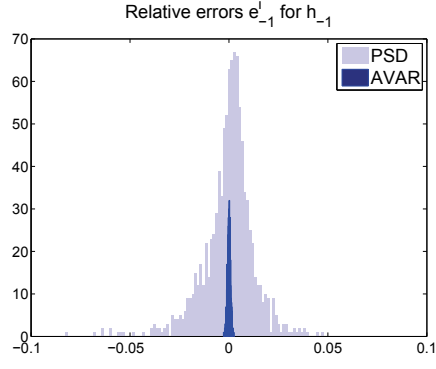

(b)

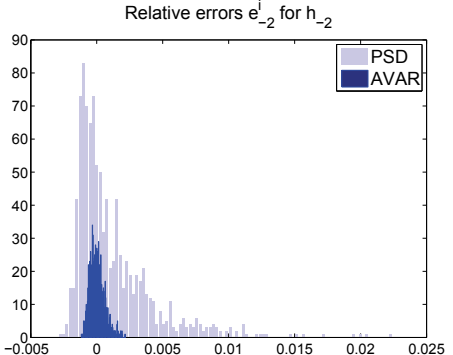

(c)

Fig. 2. Distribution of the relative estimation errors for $h_{0}$ (a), $h_{-1}$ (b), and $h_{-2}$ (c) coefficients using AVAR. The results obtained from direct estimation using periodogram are also presented which are denoted as PSD in the above figures.

noise can be thermal noise from the antennas and transceiver circuit or random shadowing effects [7] of the propagation channel.

- Blue noise $(\alpha=1)$ : With a $f$ increasing law, this term is sometimes loosely used to describe a noise with minimal low frequency components. The specific origin is not well known, but one possible source is from the interference mitigation circuit which helps combat interference and improve the wireless signal quality [20].

- Violet noise $(\alpha=2)$ : This noise presents a $f^{2}$ law and is also referred to as purple noise or differentiated white noise. Our results are aligned with no previously reported evidence of its presence in RSS measurements (to the best of our knowledge).

As we will show in the experimental section, the powerlaw identities present in RSS noise components can lead to simpler analyses if they are handled correctly. We will use the Least Squares (LS) algorithm to characterise the noise components through a parametric estimation of the AVAR as a power series (7). As studied in Section II-B (see Figure 2), the estimation method based on AVAR is preferable to direct estimation in the frequency domain based on the PSD. The noise characterisation is performed by taking advantage of the one-to-one relationship between the parametrization of AVAR and the noise PSD (see (7)).

We treat RSS coloured noise components as powerlaw processes so that they can be identified and analyzed even without the details of each noise source. Usually it is not a trivial task to identify the exact origin of each noise component. For example, [20] found that the wireless signal processing module of some peripheral cards can generate coloured noise. Specifically these network chipsets automatically increase the operational signal level and carrier sense threshold in order to ensure a certain level of signal to interference and noise ratio and the successful demodulation of incoming signals. Thus their RSS measurements usually follow a random walk behaviour, but statistically drift towards higher values with the increase of running time. The AVAR tool employed in this paper is able to analyze such time dependent behaviour even if such mechanism of the inner circuit is unknown.

\section{EXPERIMENTS}

In this section, we study two groups of RSS datasets from 802.11 systems and characterize the noise components using the AVAR tool and LS algorithm. The first group was collected by using an omnidirectional antenna and contains four datasets; the second one is from Phillips et al's work and was collected using directional antennas [21]. For the sake of comparison, we have simulated reference datasets with only white noise whose mean and standard deviation are directly calculated from the data. In each figure, we show the result of the two datasets simultaneously in order to highlight the different characterisation of coloured and white noise. We also introduce the LS algorithm to fit the curve of the RSS datasets in order to obtain the corresponding coefficients $h_{\alpha}$.

\section{A. Omnidirectional Antennas}

The first group of datasets were collected by using omnidirectional antennas on both the transmitter and receiver. We set the experiments in a $4 \mathrm{~m} \times 6 \mathrm{~m}$ laboratory surrounded with brick walls. The positions of the transmitter and receiver were fixed with the distance of $100 \mathrm{~cm}$ and both of them were equipped with a WiFi interface (Gigabyte GNWI01GT). The carrier frequency (central frequency) was set to $5.22 \mathrm{GHz}$ (WLAN Channel 44) which was known and tested to be free from any adjacent frequency interferences.

The transmitter constantly broadcasts BEACON signals every $100 \mathrm{~ms}$, which were received and demodulated by the receiver. We used an off-line application to extract the RSS measurements with time stamps from the original received packets. The noise in the RSS measurements was analyzed and its components were identified and illustrated in the figures. In total, we conducted four experiments each of them including about 60 minutes of measurements (Dataset1 to Dataset4).

The coloured noise coefficients $h_{\alpha}$ of these data were calculated by the LS algorithm and are listed in Table I. Fig.3-6 show the AVAR values of the datasets with coloured noise and the artificial white noise respectively, together with the LS fitted curve. 


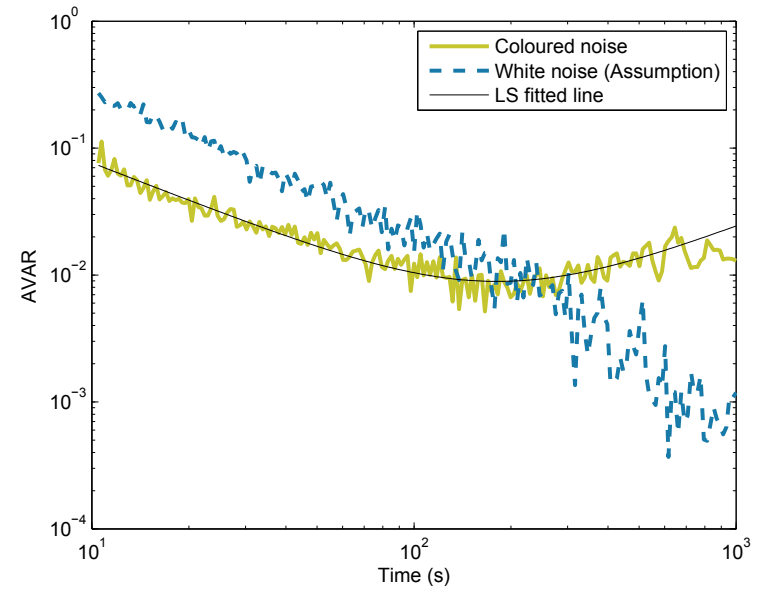

Fig. 3. AVAR and the LS fitted line of the first data set.

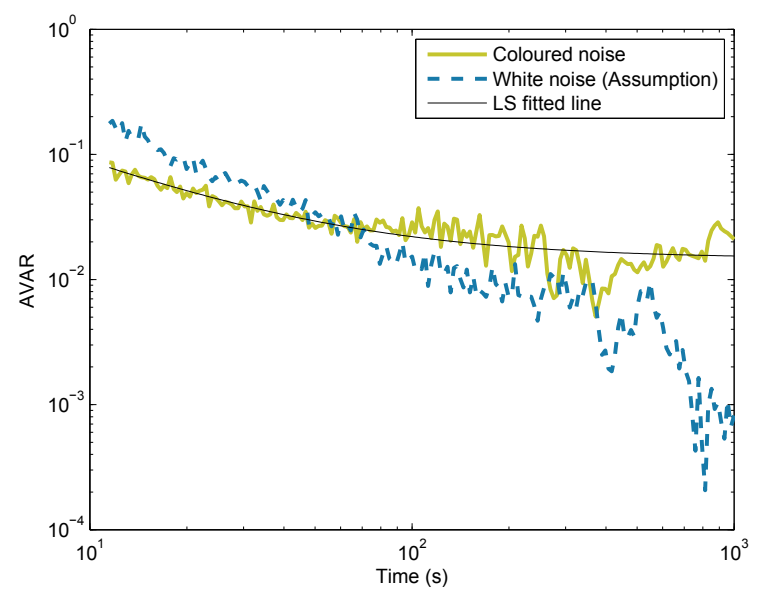

Fig. 4. AVAR and the LS fitted line of the second data set.

\section{Analysis of the results}

From Fig.3-6 and Table I, we can see that because of the existence of coloured noise, the AVAR curves of the RSS measurements do not decrease linearly with the increase of correlation time $\tau$ on the log-log diagram, whereas the ones with artificial white noise do follow this linear law. We can thus conclude that the traditional white noise assumption is inaccurate $\left(X_{\sigma}\right.$ in (2)).

Furthermore, the contributions from different noise components are stable as the results from all the four datasets have similar trends and coefficients. The three major contributions to RSS measurement noise are from Brownian $\left(h_{-2}\right)$, flicker $\left(h_{-1}\right)$ and white $\left(h_{0}\right)$ types of noise (See Table I). Theoretically we cannot exclude the other two components - $h_{1}$ and $h_{2}$, however, the overall AVAR and noise of RSS measurements is dominated by the three types mentioned before. In practice, the complexity to model and process coloured noise can be simplified [22], [23]. From the experiments, we can infer that if the transmitter system, receiver system and propagation environment remain



Fig. 5. AVAR and the LS fitted line of the third data set.

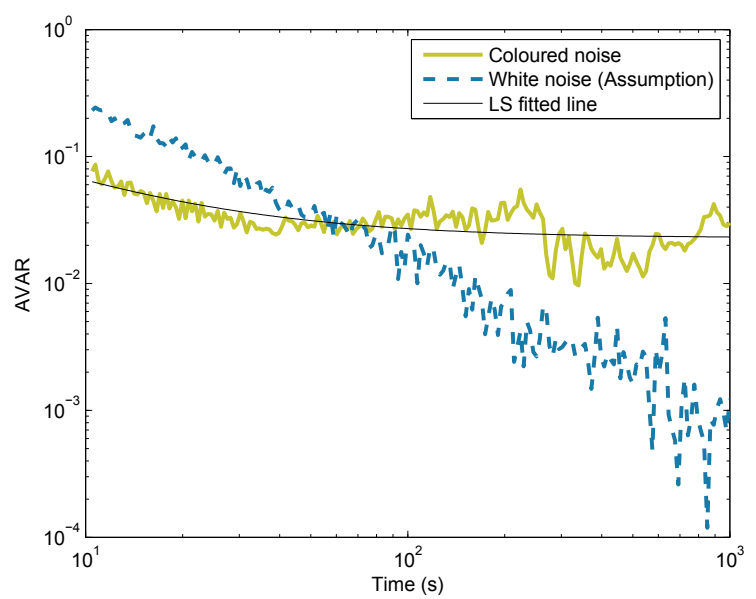

Fig. 6. AVAR and the LS fitted line of the fourth data set.

unchanged, the combination of coloured noise should be stable.

We can also see from the figures that the fluctuations in the curve become more intensive for higher $\tau$ values. The explanation for this comes from the intrinsic nature of AVAR calculation: the larger the correlation lag, the lower the number of samples available to estimate the variance [see (5)], and thus the curve becomes less smooth.

\section{B. Directional Antennas}

The next group of datasets were collected by Caleb Phillips and Eric W. Anderson from the University of Colorado [21]. In their experiments, the transmitter system used a directional antenna which was mounted to a geared triaxial head with the ability to rotate precisely. The receiving antenna was 100 feet $(\approx 30.48 m)$ away from the transmitter, fixed to its seat and omnidirectional. Both the transmitter and receiver work on the $2.4 \mathrm{GHz}$ band and have an Atheros-based MiniPCI-Express radio interference.

Different from the previous indoor experiments, the data 
TABLE I

PARAMETER $h_{\alpha}$ OF THE MEASUREMENTS FROM OMNIDIRECTIONAL ANTENNA.(THE VALUES OF $h_{1}$ AND $h_{2}$ SUGGEST THE TRIVIAL CONTRIBUTION FROM HIGHER ORDER NOISE COMPONENTS.)

\begin{tabular}{lcccc}
\hline AVAR $h$ & Dataset1 & Dataset2 & Dataset3 & Dataset4 \\
\hline$h_{-2}$ & 0.000459 & $3.169 \mathrm{e}-14$ & $2.337 \mathrm{e}-14$ & $2.287 \mathrm{e}-14$ \\
$h_{-1}$ & 0.000398 & 0.010600 & 0.020693 & 0.016444 \\
$h_{0}$ & 1.51436 & 1.46363 & 0.984316 & 0.848535 \\
$h_{1}$ & $9.999 \mathrm{e}-7$ & $1.878 \mathrm{e}-10$ & $1.060 \mathrm{e}-7$ & $9.996 \mathrm{e}-7$ \\
$h_{2}$ & $9.998 \mathrm{e}-7$ & $3.032 \mathrm{e}-14$ & $7.545 \mathrm{e}-10$ & $9.997 \mathrm{e}-7$ \\
\hline
\end{tabular}

TABLE II

PARAMETER $h_{\alpha}$ OF THE MEASUREMENTS FROM DIRECTIONAL ANTENNA.

\begin{tabular}{lcccc}
\hline AVAR $h$ & $0^{\circ}$ & $90^{\circ}$ & $180^{\circ}$ & $270^{\circ}$ \\
\hline$h_{-2}$ & 0.000075 & 0.000096 & 0.000576 & 0.000459 \\
$h_{-1}$ & 0.014009 & $8.138 \mathrm{e}-8$ & 0.155812 & $4.765 \mathrm{e}-7$ \\
$h_{0}$ & 0.017734 & 0.026985 & 0.013213 & 0.042719 \\
$h_{1}$ & $1.046 \mathrm{e}-12$ & $9.999 \mathrm{e}-7$ & $9.997 \mathrm{e}-7$ & $9.998 \mathrm{e}-7$ \\
$h_{2}$ & $2.402 \mathrm{e}-14$ & $9.997 \mathrm{e}-7$ & $9.998 \mathrm{e}-7$ & $9.999 \mathrm{e}-7$ \\
\hline
\end{tabular}

were collected in an open flat flood plain which is free from obstacles in all directions and radio interferences at the $2.4 \mathrm{GHz}$ band. The researchers recorded RSS measurements received at each 5 degree azimuth position. In order to control the length of this article, we picked the four typical azimuth angles - 0,90,180 and 270 degrees, and processed the datasets at these directions.

Each of these datasets contains $80 \mathrm{~s}$ of measurements. Comparing with the datasets collected in the previous experiment, the length is shorter. The AVAR calculations only cover a short correlation time interval, e.g. up to 80s. Nevertheless, the effects of coloured noise can still be observed from the results. Fig.7 - Fig.10 show the RSS measurements and the corresponding AVAR values for the transmitting antenna at the azimuth direction of $0,90,180$ and 270 degrees, together with the comparable datasets with artificial white noise. The coefficients of the five types of noise components are listed in Table II.

\section{Analysis of the results}

From the results we can see that the antenna direction not only affects the receiving signal strength which is common known, but also significantly changes the variation and noise components. Antenna theory suggests that the main lobe (0-degree) of a directional antenna provides the strongest RSS values, the two side lobes (90- and 270degrees) are less powerful in emitting energy and the back lobe (180-degrees) is obviously the weakest.

Fig. 7 (0-degree) shows that this dataset has higher average strength than the ones from other directions. Furthermore, the RSS values are more stable with the change of time. The largest variation observed corresponds to the dataset received at 180-degrees (Fig. 9), with higher noise content than the others.

The variation of noise combination from different di- rections shows an interesting pattern. A possible reason is that when the received signal is strong, some of the noise components are dominated by the main one, and thus the AVAR of RSS measurements demonstrates outstanding white noise features (the AVAR curve has a linear decreasing rate). However, when the correlation time becomes greater, the random walk noise is averaged by (5) and becomes less significant. Then, the other types of noise stand out, e.g. Brownian and flicker noise. This trend becomes more obvious when the signals are not received from the main lobe. Especially for the data received at 180degrees, its pink noise component is significantly stronger than for the other datasets. The fitted coefficients $h$ also show such behaviour. Similar to omnidirectional antennas, the curves of this experiment become less smoothed with the increase of correlation time because of less samples for the average function.

This experiment suggests that in order to mitigate the noise effects (both white and coloured noise), we should try to receive as high RSS as possible, thus the main lobe of directional antenna should always be used. However, in a mobile scenario, precise direction between transmitter and receiver is usually difficult to achieve. If there are only directional antennas, we would observe large strength variations at the receiving power. Practical mobile systems either use omnidirectional antenna or simulate an omnidirectional antenna by combining several directional antennas [24]. Therefore, for the directional antenna, we will only consider the noise components from the data collected at near 0degrees (Table II), which have two major members: Pink $\left(h_{-1}\right)$ and white $\left(h_{0}\right)$, and one minor component $\left(h_{-2}\right)$.

\section{DISCUSSION}

This paper focuses on the analysis of noise with distinctive AVAR or PSD. Results from our experiments using an omnidirectional receiving antenna, (Fig.3-6), show that if the transmitter, receiver and environment remain the same, the combination of noise components is stable, and thus they can be modelled by the coefficients $h_{\alpha}$. Such treatment provides a tangible method in modelling and processing each noise component and their various combinations. This method provides a good tool for designing the network module of knowledge management systems. For example, if a mobile station of a knowledge management system is using omnidirectional antennas, the coloured noise components can be cancelled based on their coefficients. The details of hardware implementation is beyond this paper and will be provided in our follow-on work.

We experimentally study the noise components using 802.11 signals in this paper, however, the proposed methodology can be directly applied to analyze other wireless signals whose strength can be modelled by the propagation model (2). As long as the noise of wireless signals follow the power-law property, similar techniques can be developed to identify and mitigate the corresponding coloured noise components. The methodology of this paper provides 


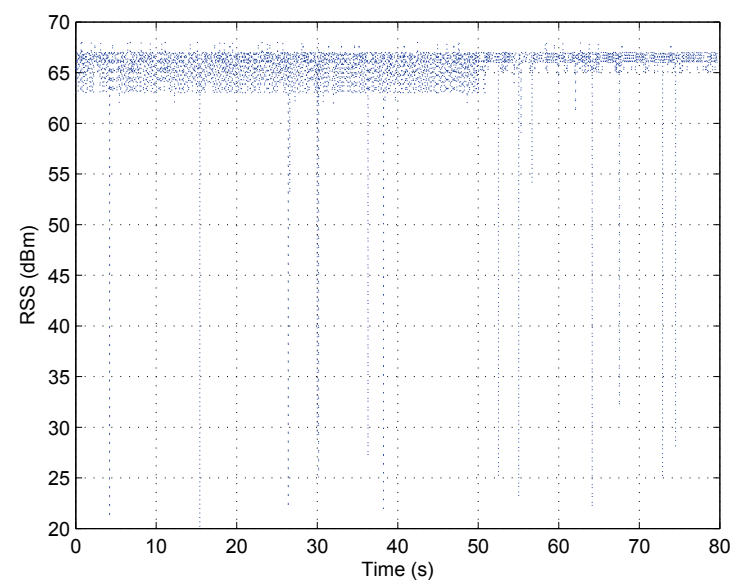

(a) RSS measurements.

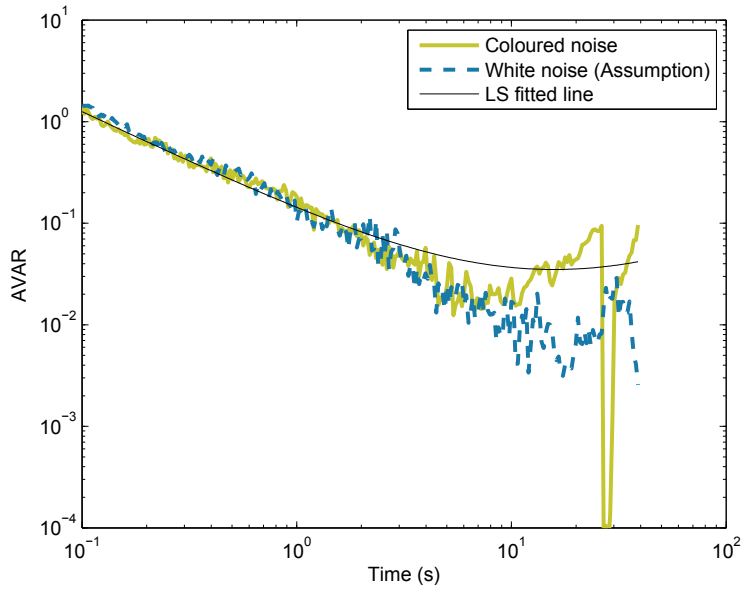

(b) AVAR.

Fig. 7. The RSS measurements and AVAR from the directional antenna system at its 0 degree direction.

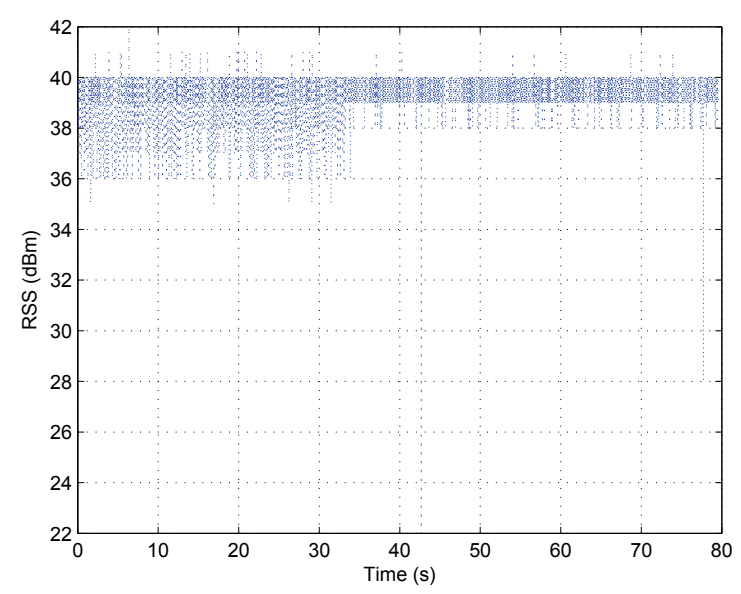

(a) RSS measurements.

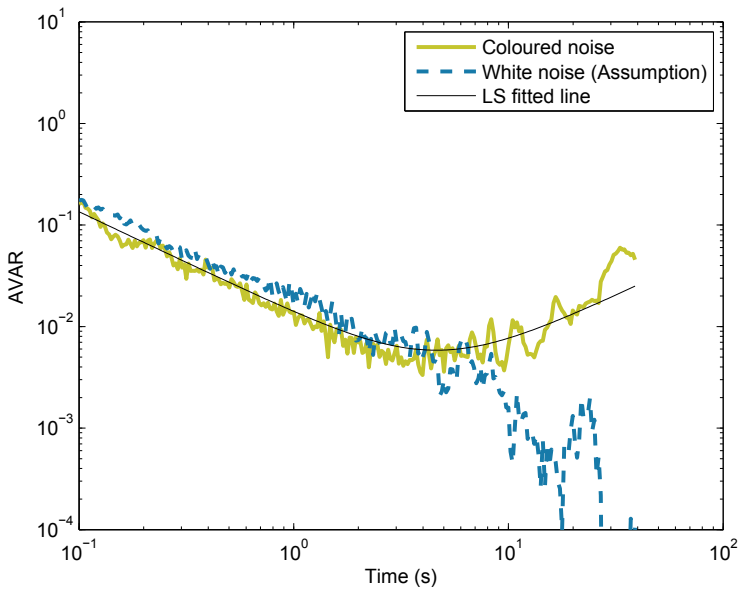

(b) AVAR.

Fig. 8. The RSS measurements and AVAR from the directional antenna system at its 90 degree direction.

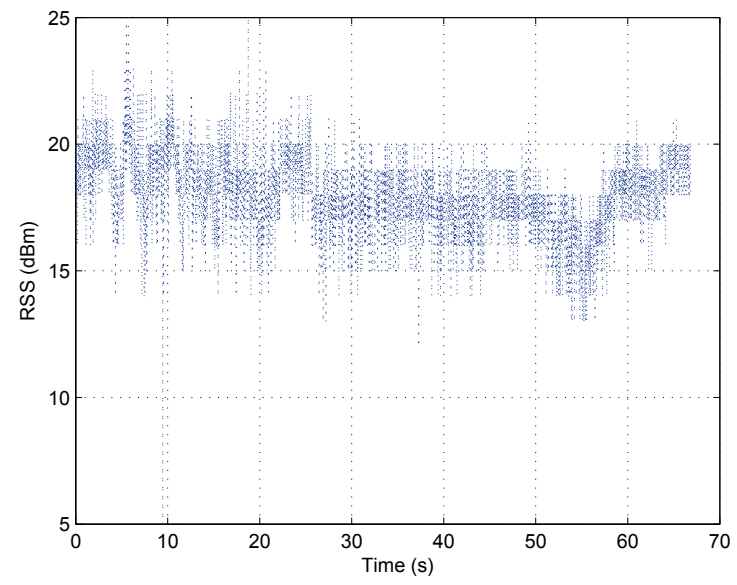

(a) RSS measurements.

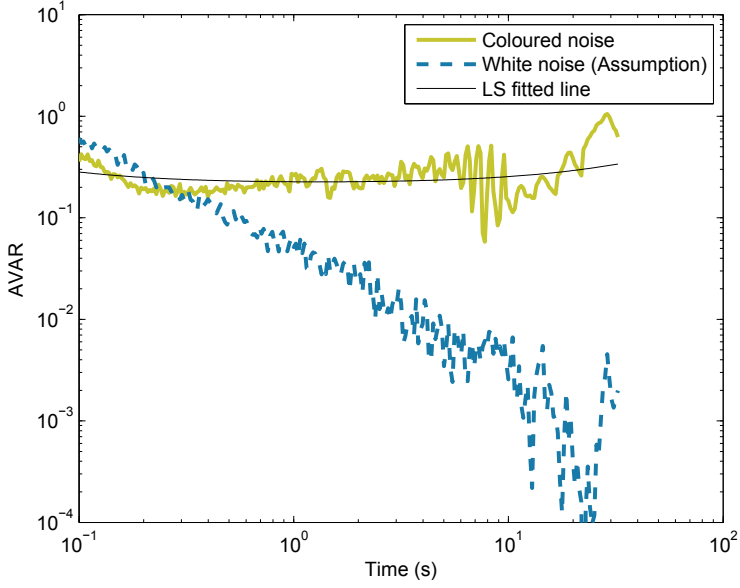

(b) AVAR.

Fig. 9. The RSS measurements and AVAR from the directional antenna system at its 180 degree direction. 


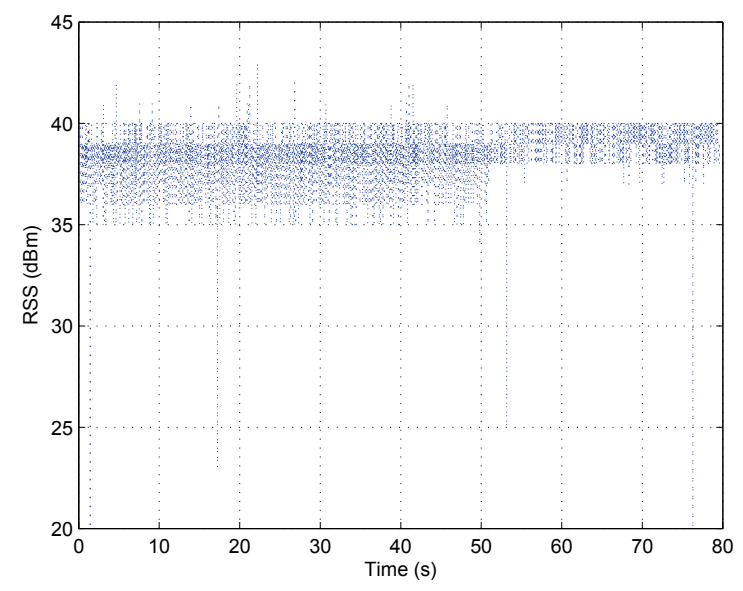

(a) RSS measurements.



(b) AVAR.

Fig. 10. The RSS measurements and AVAR from the directional antenna system at its 270 degree direction.

a tangible solution for developing knowledge management system infrastructure

\section{CONCLUSION}

In order to develop advanced networking infrastructure for future knowledge management systems, this paper studied the noise components of wireless signals that may affect the network performance. We first introduced the radio frequency signal propagation model and analyzed the noise term in detail. Theoretically, five noise components were defined, however, our experiments found only three major coloured noise components. The estimated coefficients from experiments, which denote the contributions of each component, can be clearly identified using the proposed methodology. The analyzing method and results are useful in practical scenarios requiring accurate modelling and processing of RSS.

\section{REFERENCES}

[1] A. Wadhwa, U. Madhow, J. P. Hespanha, and B. M. Sadler, "Following an RF trail to its source," in Proc. of the 49th Annual Allerton Conf. on Comm., Contr., and Computing, Sep. 2011.

[2] I. Guvenc, C. T. Abdallah, R. Jordan, and O, "Enhancements to RSS based indoor tracking systems using Kalman filters," GSPx International, 2003.

[3] T. Belis and A. Doulamis, "Distributed multimedia metadata tracking and management: An ontology-based approach with use of rss," in Systems, Signals and Image Processing, 2009. IWSSIP 2009. 16th International Conference on, June 2009, pp. 1-4.

[4] H. Xiao, M. Yuan, and Y. Yan, "A real-time event driven architecture for management information system," in Industrial Technology, 2008. ICIT 2008. IEEE International Conference on, April 2008, pp. 1-4.

[5] H. Friis, "A note on a simple transmission formula," in Pro. IRE. 34, 1946, pp. 254-256.

[6] D. C. Cox, R. R. Murray, and A. W. Norris, "800 MHz attenuation measured in and around suburban houses," Bell Labs Tech. J., vol. 63, pp. 921-954, 1984.

[7] T. S. Rappaporat, Wireless Communications - Principle and Practice. Prentice-Hall, 1996.

[8] J. G. Proakis, Digital communications, 4th ed. New York: McGrawHill, Inc., 2001.
[9] A. Goldsmith, Wireless communications. Cambridge University Press, 2005.

[10] K. Pahlavan and A. Levesque, Wireless Information Networks, ser. Wiley Series in Telecommunications and Signal Processing. Wiley, 2005.

[11] R. Zekavat and R. Buehrer, Handbook of Position Location: Theory, Practice and Advances, ser. IEEE Series on Digital \& Mobile Communication. John Wiley \& Sons, 2011.

[12] P. Hänggi and P. Jung, "Colored noise in dynamical systems," Advances in Chemical Physics, vol. 89, pp. 239 - 326, 1995.

[13] D. Allan, "Statistics of atomic frequency standards," Proc. IEEE, vol. 54, no. 2, pp. $221-230$, Feb. 1966.

[14] S. Seidel and T. Rappaport, "914 MHz path loss prediction models for indoor wireless communications in multifloored buildings," IEEE Trans. Antennas Propag., vol. 40, no. 2, pp. 207-217, Feb. 1992.

[15] L. C. Ng, "On the application of Allan variance method for ring laser gyro performance characterization," Oct., p. 29, 1993.

[16] G. M. Ljung and G. E. P. Box, "On a measure of lack of fit in time series models," Biometrika, vol. 65, no. 2, pp. 297-303, 1978.

[17] I. S. 1999, "IEEE standard definitions of physical quantities for fundamental frequency and time metrology - random instabilities," IEEE Std 1139-1999, pp. 1-36, 1999.

[18] F. Vernotte, E. Lantz, J. Groslambert, and J. Gagnepain, "Oscillator noise analysis: multivariance measurement," IEEE Trans. Instrum. Meas., vol. 42, no. 2, pp. 342-350, Apr 1993.

[19] D. Allan, "Should the classical variance be used as a basic measure in standards metrology?" IEEE Trans. Instrum. Meas., vol. IM-36, pp. 646-654, 1987.

[20] S. Robitzsch, L. Murphy, and J. Fitzpatrick, "An analysis of the received signal strength accuracy in 802.11a networks using atheros chipsets: A solution towards self configuration," in IEEE GLOBECOM Workshops (GC Wkshps). IEEE, Dec. 2011, pp. 1429-1434.

[21] C. Phillips and E. W. Anderson, "CRAWDAD data set cu/antenna (v. 2009-05-08)," Downloaded from http:// crawdad .cs .dartmouth .edu/cu/antenna, May 2009.

[22] A. Bryson and D. Johansen, "Linear filtering for time-varying systems using measurements containing colored noise," IEEE Trans. Autom. Control, vol. 10, no. 1, pp. 4 - 10, Jan. 1965.

[23] R. G. Brown and P. Y. C. Hwang, Introduction to Random Signals and Applied Kalman Filtering, P. Y. C. Hwang, Ed. John Wiley \& Sons, 1997, vol. 2, no. 4.

[24] A. Molisch, Wireless Communications, ser. Wiley - IEEE. John Wiley \& Sons, 2012. 